\title{
Survey of Chromites Fields Adjacent Rocks Loosening in Case of Their Uncontrolled Caving
}

\author{
Haini-Kamal Kassymkanova, Gulnar Jangulova, Jeniskul Bastaubaeva ", Gulbanu Baidauletova \\ Al-Farabi Kazakh National University, Almaty, Republic of Kazakhstan \\ *Corresponding Author: kzjenis@mail.ru
}

Copyright (C) 2013 Horizon Research Publishing All rights reserved.

\begin{abstract}
Control and regulation of the formation behavior while using the uncontrolled caving method require thorough investigation of the formation properties and structure, its geological, geostructural and geotechnical parameters as well as geomechanical peculiarities of rock pressure generation and its effect in the course of ore deposits mining. The most indicative are the mining plants of Donskoy Mining and Concentration Complex, which operate the uncontrolled caving method for mining of thick ore deposits at the chromite ore fields. The fields of South-Kem pirsay chromite-bearing block are the second largest fields comparing to Republic of South Africa and Zimbabwe. Prospectivity and extent of the operations at the fields could be described by the rate of production and its development: "Molodyozhnaya" mine of 2.0 million tons annual output is the world largest underground mining of chromite ore.Currently mining of ore deposits at Donskoy chromites fields is carried out at rather significant depths (of 500 and more meters), the second working of subjacent strata is carried out as well.Along with the extension of second working areas under the specified conditions, carrying out of the large-scale development operations and pre-production development as well as generation of closed extraction mines, there is the necessity to ensure more rational regulation of the rock pressure, provision for safe structural components of the mining system, selection of the methods and means for mine workings fixing and, finally, provision of efficient and reliable system of ore deposits mining. In applying this system it is critical to determine the caving zone sizes, which could be calculated quite easily depending on the ore body parameters, direction and sequence of its reserves development, whereas its height would depend on the extent of the increase of the caving rock block volume due to its loosening. This article outlines the results of laboratory tests on determination of the volume of loose rocks, which is calculated on the basis of the loosening factor $\left(\mathrm{k}_{\mathrm{p}}\right)$ that represents the "...ratio of the rock volume in loose (bulk) state to its volume as the solid block...".
\end{abstract}

Keywords Geomechanical Processes, Loosening Factor, Method of Testing, Laboratory Testing

\section{Introduction}

Speed-up of the scientific and research progress as well as the development of Kazakhstan economy are anticipated to happen due to the increase of the resources mining efficiency along with the enhancement of working conditions and ensuring safety thereof, increase of ore reserves extraction from the subsoil and reduction of adverse impact of mining operations on the stability of the underworked formation atop of which the industrial and civil buildings are located. Materials analysis evidences the increase of negative impacts of the rock pressure. Further propagation of complicating geomechanical processes leads, as a rule, to accidents, involving personal injuries, incomplete excavation of reserves, relocation of buildings and facilities, additional expenses on abandonment. Thus it is critical to explore the essence of these phenomena and, with regard to geomechanical processes regularities, make the justified decision on the control methods of the technological mining scheme, determine all required mine engineering parameters.

In developing the flat-laying ore bodies of Donskoy chromite fields at $800-1200 \mathrm{~m}$ depth ("Millionnoye" field, ore bodies 2; "Almaz-Zhemvhuzhina", ore bodies 3, 4, 7) for which uncontrolled caving of ore is typical at the relatively small ore bodies sizes in terms of depth $(80-150 \mathrm{~m})$ and thickness of 40-100 m, it is very probable that the caving zone will not outcrop, and the roof arch will stop propagating as soon as it reaches its certain height due to "self-cleavaging" with loose rocks. Intensive fracturing of the rock massif would contribute to this, when superincumbent rock caving follows the uncontrolled caving of the ore massif without significant retention and formation of the natural arch according to the generally accepted concept (when the rock caving process may cease providing availability of free space under this arch).

Distribution of the rock pressure on permanent mine openings, development and preparatory workings would depend at most and basically, on the area and height of caving zone, which will be generated in the course of second working. Area of the caving zone is calculated rather easily depending on the ore body parameters, direction and sequence of its reserves development, whereas its height would depend on the extent of the increase of the caving rock block volume due 
to its loosening. Increase in the volume of loose rocks is typically calculated on the basis of the loosening factor $\left(\mathrm{k}_{\mathrm{p}}\right)$ that represents the "...ratio of the rock volume in loose (bulk state to its volume as the solid block..." [1]. This factor describes occurrence of cavities in the caved aggregation. Authors of some published papers define such cavities as "porosity", but reasoning from the definition that "... porosity - is the total relative (in \%) volume of pores found in the mine rock..." [1], then admittedly this property describes primarily the material of the rock massif itself but not the fact that its structure and the rock block porosity (structural block) would not change upon its separation from the undisturbed massif. We will not consider this kind of porosity, that is why further we will operate the term "cavitation" of loose rock, which would mean the total relative volume of cavities (in \%) in the loose rock, which were formed during the cavitation process.

Cavitation value is expressed by the ratio (in \%) of difference in volumes of caved and non-caved rocks to the caved rock volume.

As the part of analytical study of the density and porosity (equivalent of cavitation to us) of the granular environment, the author of the paper [2] has idealized such environment as an infinite number of uniform spheres, regularities arranged layer-wise according to five arrangement patterns. The least dense pattern is cubic ("sphere on sphere"), which has porosity of $47.64 \%$ (conventionally $k_{p}=1.91$ ), the most dense - are pyramidal and tetrahedral - they have porosity of $25.95 \%$ (conventionally $\mathrm{k}_{\mathrm{p}}=1.35$ ). Herewith, it is analytically confirmed that there is no dependence of the arrangement density and porosity (cavitation) from the spheres diameter, providing the uniformity of spheres in this environment. It should be clarified that the author does not loosen certain initially monolithic environment but creates it from the spheres of equal diameter, thus we represent, in this case, the loosening factor as the conventional parameter, in order to enable its future comparison with the results of our studies.

\section{Methodology}

This very paper [2] describes the examples (with reference to other researches), where the density of granular environment was increasing due to mixing of various grains fractures. So, for example, there have been prepared the fractures of wash gravel grains - of the coarse, medium and fine size - with average values of diameters ratios equaling to 50.5:8:1. Aggregate composed of coarse grains only has been compacted up to porosity of $37.7 \%$, those from medium grains - up to $37.7 \%$, those from fine grains - up to $42.5 \%$. According to our calculations, the conventional $\mathrm{k}_{\mathrm{p}}$ for these fractures makes 1.605 and 1.739 correspondingly. Two-component mixture of $70 \%$ of coarse and $30 \%$ of medium grains (diameter ratio is 6.3) demonstrated the porosity of $26.2 \%$ ( i.e. $k_{p}=1,355$ ), in the mixture of $70 \%$ of coarse and $30 \%$ of fine grains (diameters ratio is 50.5 ) the porosity has reduced to $18.5 \%$ $\left(\mathrm{k}_{\mathrm{p}}=1,227\right)$. Basing upon the specified data it could be concluded that density and porosity of loose mixture of various sized grains depend on both the ratio of grains sizes of different fractions, which compose this mixture, and volume concentration of each fraction in the mixture.

For comparison purposes we have performed the experimental surveys on the basis of lumpy loose mass. The conditions, procedure and basic results of these laboratory experiments are given below.

To make the experiments we have used the metal vessel in the form of cylindrical tank of the following inner dimensions $\mathrm{d}=18 \mathrm{~cm}, \mathrm{~h}=20.5 \mathrm{~cm}$, volume $\mathrm{V}=5.21$. The tested material, which imitates the caved rock is loose mass of cut ceramic tile, sorted by fractions -conventionally coarse (I), medium (II), and fine (III). While soaking in water, ceramic does not practically absorbs water providing relatively short soaking time (10-15 min). The sizes of pieces in three mutually perpendicular directions vary within nearly identical ranges, which make approximately $\pm 20 \%$ of average statistical value. The length of the edge of the conventional cubic-shape piece $\left(a_{c p}\right.$ (average $)$ has been accepted as the coarseness value of loose material for each fraction [7].

Determination of $a_{c p}$ for each fraction (I,II, III) is as follows, upon its mixing, the part of its volume is separated, the lumps are calculated piece by piece, then they are all placed into the measuring tank, and overwatered to ensure that all pieces are covered with water, the volume of pieces and water is measured according to the scale, water is drained to separate tank until zero "dripping" condition is achieved, the total volume of pieces is determined by the difference of the total volume (pieces and water) $\mathrm{V}_{\text {об̆ }}$ and drained volume $\mathrm{V}_{\text {сл.в. }}$, while dividing this difference by the known number of pieces $\mathrm{N}_{\mathrm{k}}$ we can determine the average volume of one piece $v_{k}, a_{c p}$ is determined by equaling this value to the conventional cubic-shape piece and by taking the root of the value of this cubic root volume, i.e.

$$
a_{c p}=\sqrt[3]{v_{\kappa}}
$$

where $v_{\mathrm{\kappa}}=\frac{V_{\text {общ }}-V_{\text {сл.в }}}{\mathrm{N}_{\text {к }}}$

$a_{c p}$ value for each fraction has been determined according to $-N_{k}^{I}=300$ pcs., $N_{k}^{I I}=885$ pcs., $N_{k}^{I I I}=900$ pcs. During the calculation the following values have been obtained: $a_{\mathrm{cp}}^{\mathrm{I}}=12.1 \mathrm{~mm} ; a_{\mathrm{cp}}^{\mathrm{II}}=8.2 \mathrm{~mm} ; a_{\mathrm{cp}}^{\mathrm{III}}=4.0 \mathrm{~mm}$, i.e. the coarseness of pieces with regard to the fractions correlated as $3: 2: 1$.

Laboratory tests have been performed in order to establish the extent of loosening (cavitation) of the loose material and determine the loosening factor.

The first set of tests has been carried out for each fraction of loose material in two compaction states: in bulk ("loose") and compacted with agitation, impact vibration and ramming without pieces destruction ("compacted").

The second set of tests has been performed in mixtures 
composed of the above mentioned fractions of loose material providing various volumetric proportions of these fractions in the tested mixture.

The following conditions have been mandatorily observed in the course of tests:

1) surface of pieces prior to placing into the testing vessel (tank) should be wet, that is why the loose mixture has been moistened prior to placing into the tank and spread over cotton cloth, where it has been retained in this state for $10 \mathrm{~min}$.;

2) loose mass should be loaded into the testing tank to the level, above the control mark to be used for measuring of "water - loose mass" mixture volume, which would be used for calculations;

3) water level in the tank should correspond exactly to the mark at the tank walls, to be used for measuring of the total volume of "water - loose mass" mixture, which would be used for calculations [6].

Applying this testing method the volume of cavities in loose mass would be equal to the volume of poured water. No additional weighing is required. Each test should be performed tree times under identical conditions, including calculation of average values of cavitation loosening factor.

Example, Fraction I has been tested; tank volume, up to the control mark to measure "water-loose mass" mixture, is 4850 $\mathrm{cm}^{3}$ (permanent for all tests), loose mass has been preliminarily moisturized, and then slightly dried with cotton cloth.

1. Condition of the loose mass placed in the tank is conventionally - "Loose".

Poured water- $2350 \mathrm{~cm}^{3}$, i.e. volume of cavities $\mathrm{V}_{\text {пуст }}=2350$ $\mathrm{cm}^{3}$; solid volume $\mathrm{V}_{\text {тв }}=4850-2350=2500 \mathrm{~cm}^{3}$; cavitation 2350 $\frac{2350}{4850}$ $100=48,45 \%$; loosening factor $\kappa_{p}=4850 / 2500=1,94$.

2. Condition of the loose mass in the tank permanent for all tests - "Compacted".

Poured water-1835 cm , i.e. $\mathrm{V}_{\text {пуст }}=1835 \mathrm{~cm}^{3}$; $\mathrm{V}_{\mathrm{TB}}=4850-1835=3015 \mathrm{~cm}^{3}$ : cavitation: $\frac{1835}{4850} 100=37,62 \%$; $\kappa_{p}=4850 / 3015=1,61$.

Shrinking of the loose mass upon compaction (comparison of the tests $1 \& 2)$ is $100 \times(48,45-37,62) / 46,45=22,4 \%)$.

\section{Results}

The results of the performed tests are summarized in the table 1.

Table 1. Results of laboratory tests on determination of cavitation and $\kappa_{\mathrm{p}}$ in loose material, which imitates loose rocks.

\begin{tabular}{|c|c|c|c|c|c|c|c|c|}
\hline \multirow{2}{*}{ No. } & \multirow{2}{*}{ Loose material type } & \multirow{2}{*}{$\begin{array}{l}\text { Coarseness } \\
\text { of pieces } \\
\text { (aф),m }\end{array}$} & \multirow{2}{*}{$\begin{array}{l}\text { Material condition } \\
\text { in the tank }\end{array}$} & \multirow{2}{*}{$\begin{array}{l}\text { Solid volume in } \\
\text { the tank, } \mathrm{cm} 3\end{array}$} & \multicolumn{2}{|c|}{ Cavitation } & \multirow{2}{*}{ кр } & \multirow{2}{*}{$\begin{array}{c}\text { Shrinking, } \\
\%\end{array}$} \\
\hline & & & & & cmj & $\%$ & & \\
\hline \multirow{2}{*}{1.} & \multirow{2}{*}{ Fraction I } & \multirow{2}{*}{12.1} & Loose & 2500 & 2350 & 48.45 & 1.94 & \multirow{2}{*}{22.35} \\
\hline & & & Compacted & 3015 & 1835 & 37.62 & 1.61 & \\
\hline \multirow{2}{*}{2.} & \multirow{2}{*}{ Fraction II } & \multirow{2}{*}{8.2} & Loose & 2550 & 2300 & 47.40 & 1.90 & \multirow{2}{*}{19.54} \\
\hline & & & Compacted & 3000 & 1850 & 38.14 & 1.62 & \\
\hline \multirow{2}{*}{3.} & \multirow{2}{*}{ Fraction III } & \multirow{2}{*}{4.0} & Loose & 2485 & 2365 & 48.76 & 1.95 & \multirow{2}{*}{19.67} \\
\hline & & & Compacted & 2950 & 1900 & 39.17 & 1.64 & \\
\hline \multirow{2}{*}{4.} & \multirow{2}{*}{$\begin{array}{c}\text { Fraction } И-1 / 2 \text { volume Fraction } \\
\text { II! }-1 / 2 \text { volume }\end{array}$} & \multirow{2}{*}{ - } & Loose & 2700 & 2150 & 44.33 & 1.80 & \multirow{2}{*}{$16 . .29$} \\
\hline & & & Compacted & 3050 & 1800 & 37.11 & 1.59 & \\
\hline \multirow[b]{2}{*}{5.} & \multirow{2}{*}{$\begin{array}{c}\text { Fraction I }-1 / 3 \text { volume Fraction } \\
\text { II }-1 / 3 \text { volume Fraction III }-1 / 3 \\
\text { volume }\end{array}$} & & Loose & 2740 & 2110 & 43.52 & 1.77 & \multirow[b]{2}{*}{18.52} \\
\hline & & & Compacted & 3130 & 1720 & 35.46 & 1.55 & \\
\hline \multirow[b]{2}{*}{6.} & \multirow{2}{*}{$\begin{array}{c}\text { Fraction I - } 64 \% \text { volume Fraction } \\
\text { II - } 26 \% \text { volume Fraction III } \\
-10 \% \text { volume }\end{array}$} & & Loose & 2755 & 2095 & 43.19 & 1.76 & \multirow[b]{2}{*}{21.23} \\
\hline & & & Compacted & 3200 & 1650 & 34.02 & 1.52 & \\
\hline 7. & $\begin{array}{c}\text { Fraction I - } 50 \% \text { volume Fraction } \\
\text { II - } 25 \% \text { volume Fraction } \amalg\end{array}$ & & Compacted & 3750 & 1100 & 22.7 & $1 . .29$ & - \\
\hline
\end{tabular}




\section{Tests Results Summaries}

Cavitation and loosening factor of the loose mixture made of similar-fraction pieces do not depend on the pieces coarseness, they are nearly similar for each of three tested fractions (coarse, average and fine) and make accordingly $48-49 \%$ and $1.90-1.93$ in loose state and $37-38 \%$ and $1.59-1.62$ - in compacted state.

Mixing of the lumpy material fractions has evident impact on the values of cavitation and loosening factor towards their reduction. Adding of dry screened sand (the $4^{\text {th }}$ very fine fraction) without the increase to the total volume of the tested mixture model will significantly compact this mixture and reduce the loosening factor up to the values of 1.25-1.30.

Unconsolidated loose lumpy mass could shrink in volume up to $20 \%$ due to consequent relocation of pieces to more compact position without their destruction, which corresponds to the experimental data in the paper [3]. Such relocation of the rock pieces might take place in mine conditions when the loose rock will be displaced downwards following the broken or uncontrolled caved ore in the course of its discharge.

The results obtained in many instances correlate or are not in conflict with the results of analytical surveys published in the paper [2].

Taking into account the natural fracturing of the rock massif [4], it could be assumed that the size of the loose rock would correspond to the size of the rock massif block in the diameter, which ranges within 100-200 mm. Caving would occur without explosion load, following the ore without rock pieces falling down from large height, the caved rock mass would move during ore discharge thus the rock pieces would tend to conglomerate into more compact positions in bulk, cavities would be consequently filled with fine fractions (20-50 times smaller than structural blocks), generated due to destruction of epigenetic minerals, which fill natural fractures of the rock massif (up to $4-5 \%$ of the total cavitation volume), due to tectonic crushing zones (1-2\%), as well as smashing and abrasive wear of the structural blocks edges during their displacement in bulk ore in the course of ore discharge $(2-3 \%)$.

Assuming that $38 \%$ and 1.60 represent the initial values of cavitation and loosening factor correspondingly, the cavitation in the loose zone would make supposedly $28-30 \%$, whereas the loosening factor - $1.40-1.43$ at the final stage of second working, which include uncontrolled caving of ore and adjacent rock.

\section{Discussion and Conclusion}

According to the geologists' data [5] the adjacent rocks of Donskoy chromite fields are represented mostly by heavy silicates and olivine rocks, which are generally serpentinous up until serpentine generation. Serpentine, as an unstable mineral, could transform into agilite and chlorite (rather soft minerals) under hydrothermal influence. Due to these chemical processes and under gravitation force effect, the slow process of caved mass shrinking will keep going in the caving zone, including decrease of its cavitation loosening factor and increase of the roof arch height. These processes in Donskoy Mining and Concentration Complex are poorly known, that is why it could be assumed that in some years the cavitation of the loose mass could decrease up to $20-25 \%$, and those of loosening factor - up to $1.25-1.30$.

\section{REFERENCES}

[1] A.B. Makarov. Practical geomechanics. Manual for mining engineers. "Mining Book". Moscow, 2006.- 380p.

[2] I.B. Yedilbayev, Vozrozhdeniye (1995-2005). Almaty, 2004. $-57 \mathrm{p}$.

[3] Chrome of Kazakhstan. Reference book / Under the joint editorship of M.I. Gasyk, V.I. Grinenko, V. N. Shashkin / M. - "Metallurgy". - 2001. - P.305-306.

[4] Instruction for calculation of the loads on mine working support at the mines of Donskoy Mining and Concentration Complex. - Karaganda, 2004. - 44 p.

[5] A.D. Sashurin, Peculiarities of the mine rocks displacement process development at the fields of Donskoy Mining and Concentration Complex // Materials of the fourth international scientific and practical conference. - Khromtau. - May 23-25, 2007. - P.220-223.

[6] I.V. Baklashov, B.A. Kartoziya. Mine rocks mechanics. M.: "Publishing House of the Moscow State Mine University", 2004. $-208 \mathrm{p}$.

[7] A.N. Shashenko, E.A. Sdvizhkova, S.N. Gapayev. Deformability and failure resistance of the mine rocks massifs: Monography. Dnepropetrovsk National Mine University, 2008. 224 p. 